\title{
A Fuzzy Three Sphere and Fuzzy Tori
}

\author{
Brian P. Dolan ${ }^{a), b) *}$ and Denjoe O'Connor ${ }^{b) \dagger}$ \\ ${ }^{a)}$ Dept. of Mathematical Physics, NUI, Maynooth, Ireland \\ ${ }^{b)}$ School of Theoretical Physics, \\ Dublin Institute for Advanced Studies, \\ 10 Burlington Rd., Dublin 8, Ireland
}

September 23, 2005

\begin{abstract}
A fuzzy circle and a fuzzy 3-sphere are constructed as subspaces of fuzzy complex projective spaces, of complex dimension one and three, by modifying the Laplacians on the latter so as to give unwanted states large eigenvalues. This leaves only states corresponding to fuzzy spheres in the low energy spectrum (this allows the commutative algebra of functions on the continuous sphere to be approximated to any required degree of accuracy). The construction of a fuzzy circle opens the way to fuzzy tori of any dimension, thus circumventing the problem of power law corrections in possible numerical simulations on these spaces.
\end{abstract}

\section{Introduction}

One of the principal goals of the study of field theories on fuzzy spaces is to develop an alternative non-perturbative technique to the familiar lattice one [1]. To date, this new approach in the case of four dimensional field theories has been limited to studies of Euclidean field theory on $S^{2} \times S^{2}$ [2], $\mathbb{C P}^{2}[3]$ and $S^{4}$ [4]. All but $S^{2} \times S^{2}$ have additional complications. For

\footnotetext{
*bdolan@thphys.may.ie

†denjoe@stp.dias.ie
} 
example, $\mathbb{C P}^{2}$ is not spin but $\operatorname{spin}^{c}$ and $S^{4}$ is really a squashed $\mathbb{C P}^{3}$ and includes many unwanted massive Kaluza-Klein type modes. Even $S^{2} \times S^{2}$ is not ideal since it has curvature effects that drop off as power corrections rather than exponentially as in the case of toroidal geometries.

The fuzzy approach does, however, have the advantage of preserving continuous symmetries such as the $S U(2)$ symmetry of a round $S^{2}$ and does not suffer from fermion doubling [5]. The advantages are gained at the cost of introducing a non-locality associated with the non-commutativity of the fuzzy sphere. There is therefore a balance of advantages and disadvantages associated with the fuzzy approach. The final decision on whether the approach has real advantages over the standard lattice approach should be determined by doing genuine simulations. For this reason Monte Carlo simulations of the fuzzy approach are now in progress. In the lattice approach non-locality is also a problem when fermions are included. So our expectation is that as far as Monte Carlo simulations are concerned the fuzzy approach will not be competitive with the lattice one until fermions are included. The approach will gain further advantages in situations where symmetries are more important. It also extends naturally to allow for supersymmetry. (see [6] where a fuzzy supersphere was constructed). So we expect the true power of the approach to emerge when supersymmetry and chiral symmetry are present in a model.

A radically different alternative to the Euclidean Monte Carlo approach becomes available once one has a fuzzy three-dimensional space. Such a space has the advantage that it allows one to develop very different nonperturbative methods, since now one can address the non-perturbative questions from a Hamiltonian point of view.

The purpose of this article is to introduce precisely such fuzzy threedimensional spaces. We will begin by presenting a fuzzy version of the circle $S_{F}^{1}$, from which one can obtain tori of arbitrary dimension. We will then present a fuzzy approximation to the three-sphere, $S_{F}^{3}$. Unfortunately, both of these spaces are still not ideal in that they involve many unwanted additional degrees of freedom which we suppress so that they do not contribute to the low energy physics. The presence of additional degrees of freedom is probably unavoidable as it seems to be the price one pays for the classical space not being a phase space. The three-sphere is also curved and hence the results obtained from studies of field theories on this space will approach those of a flat three-dimensional space with polynomial corrections. It has, however, the advantages of preserving the full $S O(4)$ symmetry of a round $S^{3}$. From the construction it seems clear that both of these spaces will also be free of fermion doubling problems.

We will restrict our focus here to scalar field theories and demonstrate how 
the unwanted degrees of freedom can be suppressed so that the limiting large matrix theory of a scalar field theory recovers field theory on the commutative spaces. We will argue that the data specifying the geometries can be cleanly specified by giving a suitable Laplace-type operator for the scalar field, which together with the matrix algebra and its Hilbert space structure gives a spectral triple.

Aside from our personal motivations, non-commutative geometry has recently become a very popular area of research from both the point of view of possible new physics in string theory and $D$-brane theory, $[7,8]$, and as a new regularisation technique in ordinary quantum field theory, [2]-[4] and [9]-[12]. In both these endeavours "fuzzy" spaces play an important rôle. Roughly speaking a fuzzy space is a finite matrix approximation to the algebra of functions on a continuous manifold, the seminal example being the fuzzy two-sphere, [13]. It has the important property of preserving the isometries of the space that it is approximating. As such the idea can serve as a source of examples related to matrix models in string theory and as a regularisation technique for ordinary quantum field theory. As a regularisation method it provides one that preserves the underlying space-time symmetries and is amenable to numerical computation.

Fuzzy spheres in dimensions other than two were analysed in [14]-[17], but the construction there was incomplete. They also advocate projecting out the unwanted modes and working with a non-associative algebra which we consider unsatisfactory. Also the case of odd spheres works very differently to that of even spheres. An alternative approach for the fuzzy four-sphere, $S_{F}^{4}$, was given in [4], based on the fact that fuzzy $\mathbb{C P}^{3}$ and $\mathbb{C P}^{1} \cong S^{2}$ are well understood [18], and, in the continuum limit, $\mathbb{C P}^{3}$ is an $S^{2}$ bundle over $S^{4}$.

In this paper we show how the odd-dimensional fuzzy spheres $S_{F}^{1}$ and $S_{F}^{3}$ can be extracted from the matrix algebras associated with the fuzzy complex projective spaces $\mathbb{C P}_{F}^{1}$ and $\mathbb{C P}_{F}^{3}$. An alternative approach to obtaining a finite approximation to $S^{3} \cong S U(2)$, based on conformal field theory, was presented in [19], however, in this approach it is unclear how the unwanted modes are to be suppressed. Our method uses a similar suppression mechanism to that used for $S_{F}^{4}$ in [4]. Although there is no closed finite dimensional matrix algebra for $S_{F}^{N}$ unless $N=2$, the relevant degrees of freedom when $N=1$ and $N=3$ are contained in the matrix algebras for $\mathbb{C P}^{1}$ and $\mathbb{C P}^{3}$ respectively. One can therefore obtain functional integrals for field theories over $S_{F}^{1}$ and $S_{F}^{3}$ by starting with functional integrals over $\mathbb{C P}_{F}^{1}$ and $\mathbb{C P}_{F}^{3}$ and then suppressing the unwanted modes so that they do not contribute to the functional integral. Because of the high degree of symmetry inherent in the construction, the unwanted modes can be suppressed simply by using appropriate quadratic Casimirs in the Laplacian. In this way we by-pass 
the problems associated with the fact that the algebra of matrices associated with functions on the sphere does not close on the sphere, but necessarily lifts into the enveloping complex projective space. In a similar fashion we expect that when a Hamiltonian approach to field theory is developed using these spaces the unwanted modes will cause no difficulties since they can be made arbitrarily difficult to excite.

The paper is organized as follows. In section 2 we summarize how a given geometry is captured in the fuzzy approach. Section 3 then gives our construction of a fuzzy circle, $S_{F}^{1}$. Section 4 summarises the construction of $S_{F}^{4}$ presented in [4] and in section 5 we present our fuzzy three-sphere, $S_{F}^{3}$. Section 6 gives an alternative construction of $S_{F}^{3}$ which lends itself to a generalisation to $S_{F}^{N}$ for any $N[20]$.

\section{Encoding the geometry of a fuzzy space}

Fröhlich and Gawędzki [19] (following Connes, [21]) have demonstrated that the abstract triple $\left(H, \mathcal{A}, \Delta_{\gamma}\right)$, where $H$ is the Hilbert space of square integrable functions on the manifold $\mathcal{M}$, with Laplace-Beltrami operator $\Delta_{\gamma}, \gamma$ being the metric, and $\mathcal{A}=\mathcal{C}^{\infty}(\mathcal{M})$ is the algebra of smooth bounded functions on $\mathcal{M}$, captures a topological space together with its metrical geometry.

In a similar fashion one can specify a fuzzy space, $\mathcal{M}_{F}$, as the sequence of triples

$$
\mathcal{M}_{F}:=\left(H_{L}, \operatorname{Mat}_{d_{L}}, \Delta_{L}\right)
$$

parameterized by $L$, where $H_{L}=\mathbb{C}^{d_{L}^{2}}$ is the Hilbert space acted of the complete matrix algebra $\mathrm{Mat}_{d_{L}}$ of dimension $d_{L}^{2}$ with inner product $\langle M, N\rangle=$ $\frac{1}{d_{L}} \operatorname{Tr}\left(M^{\dagger} N\right)$ and $\Delta_{L}$ is a suitable Laplacian acting on matrices. One can readily extract information such as the dimension of the space from these data. The Laplacian comes with a cutoff and so the dimension can be read from the growth of the number of eigenvalues.

The data contained in the triple $\left(H, \mathcal{A}, \Delta_{\gamma}\right)$ are precisely the data that go into the Euclidean action for a scalar field theory on the space $\mathcal{M}$ and hence specifying the scalar action is a convenient method of prescribing these data.

In the fuzzy approach the algebra will always be a matrix algebra and we will retain the Hilbert space inner product specified above so the only data from the triple, $\left(H_{L}, \mathrm{Mat}_{d_{L}}, \Delta_{L}\right)$, remaining to be supplied are the permitted matrix dimensions, $d_{L}$ and a realization of the Laplacian, $\Delta_{L}$. Once this information is given the fuzzy geometry is specified.

Though it may be convenient to give a map to functions this is not necessary. Once the Laplacian is given its eigenmatrices and spectrum can be used to provide such a map if needed. Suppose for example that the spectrum of 
$\Delta_{L}$ is identical to that of $\Delta_{\gamma}$ up to some cutoff and a complete set of eigenmatrices is given by $\hat{\Psi}_{\lambda}$ with the corresponding commutative eigenfunctions being $\Psi_{\lambda}$, then the symmetric symbol-map $\mathrm{D}$ given by

$$
\mathrm{D}=\sum_{\lambda}^{d_{L}^{2}} \Psi_{\lambda} \hat{\Psi}_{\lambda}
$$

provides a map to functions with

$$
f_{M}=\frac{1}{d_{L}} \operatorname{Tr}(\mathrm{D} M)
$$

the function corresponding to the matrix $M$. By construction the map has no kernel and the symbol-map induces a $*$ product on functions given by

$$
f_{M} *_{\mathrm{D}} f_{N}=\frac{1}{d_{L}} \operatorname{Tr}(\mathrm{D} M N)
$$

which represents matrix multiplication in terms of an operation on the image functions. The $*$ product depends on $\mathrm{D}$, a different but equivalent one could be obtained by giving a nonzero weighting $c_{\lambda}(L)$ to the different terms in the sum (2). In the case of $\mathbb{C P}^{N}$ a particular choice of the $c_{\lambda}(L)$ will give the diagonal coherent state prescription ${ }^{1}$ as discussed in [18].

If the symbol-map (2) has the property that

$$
\Delta_{\gamma} f_{M}=\frac{1}{d_{L}} \operatorname{Tr}\left(\mathrm{D} \Delta_{L} M\right)
$$

where $\Delta_{\gamma}$ is a natural Laplacian for the space to be approximated, then the spectrum of the fuzzy space will be precisely a cutoff version of that of the commutative space $\mathcal{M}$. This is precisely what happens in the case of $\mathbb{C P}_{F}^{N}$, see $[18]$.

However, it is convenient to extend the definition of fuzzy space to the case where the spectrum coincides for low-lying eigenvalues, but deviates for a family of eigenvalues that can be given arbitrarily high value and which correspond to degrees of freedom that have no counterpart in the commutative space $\mathcal{M}$. This allows us to obtain fuzzy approximations to additional spaces - in particular, as we will see, to tori and the three sphere.

\footnotetext{
${ }^{1}$ In the case where the symbol-map is the projector of coherent states the function $f_{M}$ is referred to as the covariant symbol of the matrix $M$ and since the coefficients $c_{\lambda}(L)$ are not one it will differ from the corresponding contravariant symbol, see Berezin [22]. The symbol-map is referred to as symmetric when its covariant and contravariant symbols are equal and coincides with the case of $c_{\lambda}(L)=1$.
} 
If one takes the Euclidean quantum field theory point of view then the desired geometry appears as that associated with the accessible configurations of the field theory and the deviations are suppressed in a probabilistic fashion.

A successful method of suppressing the unwanted modes would be to add to the scalar action a term $S_{I}[\Phi]$ which is non-negative for any $\Phi$, zero only for matrices that correspond to functions on $\mathcal{M}$, and positive for those that do not. The modified action would therefore be of the form $S[\Phi]+h S_{I}[\Phi]$. The parameter $h$ should be chosen to be large and positive. The probability of any given matrix configuration then takes the form

$$
\mathcal{P}[\Phi]=\frac{\mathrm{e}^{-S[\Phi]-h S_{I}[\Phi]}}{Z}
$$

where

$$
Z=\int d[\Phi] \mathrm{e}^{-S[\Phi]-h S_{I}[\Phi]}
$$

is the partition function of the model. If the prescription is to work for free field theories, then $S_{I}[\Phi]$ should be at most quadratic in $\Phi$. This can then be thought of as a modification of the Laplacian in the triple (1).

Furthermore the problem of UV/IR mixing in scalar theories can be removed by including a higher derivative operator in the quadratic term of the field theory such that it renders all diagrams finite when the matrix size is sent to infinity. With such a prescription since each diagram has a limiting commutative value in the large matrix limit each diagram must take this value and hence no UV/IR mixing can occur. The prescription of sending the matrix size to infinity and sending the coefficient of the irrelevant higher derivative operator to zero do not commute. This prescription of adding an irrelevant operator to the action is simpler than the normal ordering prescription proposed in [23] and works for any dimension.

From the above discussion it should be clear that the entire problem of constructing a fuzzy approximation to a space is the problem of giving a suitable prescription for the matrix Laplacian.

\section{Approximating a circle from a fuzzy sphere}

Consider the finite matrix algebra representation of the fuzzy sphere $S_{F}^{2}$ [13]. The algebra of $(L+1) \times(L+1)$ matrices, which will be denoted by $\mathrm{Mat}_{L+1}$, has the same dimension as the number of degrees of freedom in a spherical 
harmonic expansion of a function on $S^{2}$, truncated at angular momentum $L$,

$$
f_{L}(\theta, \phi)=\sum_{l=0}^{L} \sum_{m=-l}^{l} f_{l m} Y_{l m}(\theta, \phi) .
$$

That is

$$
\sum_{l=0}^{L}(2 l+1)=(L+1)^{2} .
$$

The precise identification between a matrix $\Phi \in \mathrm{Mat}_{L+1}$ and a cut-off function $f_{L}(\theta, \phi)$, as discussed in the preceding section, is not unique, but the possible maps can be given in terms of coherent states or the symmetric symbolmap D of (2), and the resulting product of functions is non-commutative for finite $L$. It is crucial to our construction that only maps for which the product of functions becomes commutative in the limit $L \rightarrow \infty$ be considered. The symbol-map (2) associates orthonormal $(L+1) \times(L+1)$ polarisation tensors $\hat{Y}_{l m}$ with spherical harmonics $Y_{l m}(\theta, \phi)$. The conventions used here will be that

$$
\hat{Y}_{l m}=\frac{1}{\sqrt{L+1}} \hat{T}_{l m}
$$

where the polarisation tensors $\hat{T}_{l m}$ are those of [24].

The $S O(3)$ symmetric Laplacian, $\mathcal{L}^{2}$, on the fuzzy sphere acts on matrices $\Phi$ and is represented by the second order Casimir corresponding to the adjoint action of the angular momentum generators $L_{i}$ in the $(L+1) \times(L+1)$ representation:

$$
\mathcal{L}^{2} \Phi=\left[L_{i},\left[L_{i}, \Phi\right]\right] .
$$

Hence the action can be taken to be

$$
S[\Phi]=\frac{1}{L+1} \operatorname{Tr}\left(\frac{1}{2} \Phi^{\dagger} \mathcal{L}^{2} \Phi+V(\Phi)\right)
$$

for some scalar potential $V(\Phi)^{\dagger}=V(\Phi)$, which is assumed to be bounded below. This action can then be used in a partition function which involves ordinary integration over $(L+1)^{2}$ degrees of freedom

$$
Z=\int \mathcal{D} \Phi \mathrm{e}^{-S[\Phi]}
$$

The probability distribution for field configurations is then

$$
\mathcal{P}[\Phi]=\frac{\mathrm{e}^{-S[\Phi]}}{Z}
$$


where $S[\Phi]$ given is by (12). This probability distribution is associated with the geometry $\left(H_{L}, \mathrm{Mat}_{L+1}, \mathcal{L}^{2}\right)$ which specifies a round fuzzy sphere. The field theory with quadratic potential, however, suffers from UV/IR mixing problems $[23,25]$. If we add the term $a \mathcal{L}^{4}$ to the Laplacian and use the triple $\left(H_{L}\right.$, Mat $\left._{L+1}, \mathcal{L}^{2}+a \mathcal{L}^{4}\right)$ the UV/IR mixing problem is removed and we recover a field theory on the commutative $S^{2}$ in the infinite matrix size limit. The parameter $a$ can finally be sent to zero with the result that the critical value of the mass parameter will be sent to infinity. The process of taking the large matrix limit and sending $a$ to zero do not commute. To obtain the commutative theory on the sphere the matrix size must be sent to infinity for non-zero $a$.

There is no finite matrix approximation to the algebra of functions on $S^{1}$. Nevertheless, the degrees of freedom relevant to a circle are certainly contained in $\mathrm{Mat}_{L+1}$. Focusing on the top harmonic in (8), with $l=L$, the $Y_{L m}$ contain all $-L \leq m \leq L$ and thus reproduce functions on the circle as $m \rightarrow \infty$. This implies that the partition function and correlation functions for a field theory on a circle can be extracted from that of the fuzzy sphere by suppressing all the modes with $l<L$ in (13). One way of achieving this is to penalise modes with $l<L$ by giving them a large positive weight in the action. To this end we modify the action (12) to

$$
S_{h}[\Phi]=\frac{1}{L+1} \operatorname{Tr}\left\{\frac{1}{2} \Phi^{\dagger}\left[L_{3},\left[L_{3}, \Phi\right]\right]+\frac{h}{2} \Phi^{\dagger}\left(L(L+1)-\mathcal{L}^{2}\right) \Phi+V(\Phi)\right\} .
$$

All modes with $l<L$ now have the wrong sign for $\mathcal{L}^{2}$ and, when $h$ is very large, are heavily penalised in the partition function (13), contributing nothing as $h \rightarrow \infty$. In this limit only the modes with $l=L$ remain and these have the correct sign for their kinetic energy, because the term linear in $h$ vanishes on these and only these modes. The 'wrong sign' for the $\mathcal{L}^{2}$ contribution to the kinetic energy here is analogous to an anti-ferromagnetic coupling in a lattice theory and just as in the lattice theory with an antiferromagnetic coupling the action here is also bounded below. That the action remains bounded from below is intimately related to the fact that there is an ultraviolet cutoff in the model and therefore a maximum eigenvalue for the Laplacian or equivalently a shortest wavelength.

To see that the commutative algebra of functions on $S^{1}$ is recovered in the $l=L$ sector of the fuzzy sphere as $L \rightarrow \infty$, we first decompose the matrix $\Phi$ using the basis of polarisation tensors:

$$
\Phi=\sum_{l=0}^{L} \sum_{m=-l}^{l} \Phi_{l m} \hat{Y}_{l m} .
$$


In our conventions (10) the commutator of the polarisation tensors is given by (see e.g. [24] page 191, equation (46))

$$
\begin{array}{r}
{\left[\hat{Y}_{l_{1} m_{1}}, \hat{Y}_{l_{2} m_{2}}\right]=\sqrt{\frac{\left(2 l_{1}+1\right)\left(2 l_{2}+1\right)}{L+1}} \sum_{l=0}^{L}(-1)^{L-l}\left\{1-(-1)^{l_{1}+l_{2}+l}\right\}} \\
\times\left\{\begin{array}{ccc}
l_{1} & l_{2} & l \\
L / 2 & L / 2 & L / 2
\end{array}\right\} C_{l_{1} m_{1}, l_{2} m_{2}}^{l m} \hat{Y}_{l m},
\end{array}
$$

where $\left\{\begin{array}{ccc}l_{1} & l_{2} & l \\ L / 2 & L / 2 & L / 2\end{array}\right\}$ are $6 j$-symbols and $C_{l_{1} m_{1}, l_{2} m_{2}}^{l m}$ are Clebsch-Gordon co-efficients. Now for large $L$

$$
\left\{\begin{array}{ccc}
l_{1} & l_{2} & l \\
L / 2 & L / 2 & L / 2
\end{array}\right\} \approx \frac{1}{\sqrt{L+1}} C_{l_{1} 0, l_{2} 0}^{l 0}
$$

and $C_{l_{1} 0, l_{2} 0}^{l 0}=0$ when $l_{1}+l_{2}+l$ is odd. Thus

$$
\left[\hat{Y}_{l_{1} m_{1}}, \hat{Y}_{l_{2} m_{2}}\right] \rightarrow 0
$$

and the algebra is commutative when $L \rightarrow 0$ as promised. In particular

$$
\left[\hat{Y}_{L m_{1}}, \hat{Y}_{L m_{2}}\right] \rightarrow 0
$$

and the top harmonic alone reproduces the commutative algebra of functions on $S^{1}$ in the continuum.

To summarize we can encode the geometry specifying a fuzzy circle by the triple

$$
S_{F}^{1}:=\left(H_{L}, \operatorname{Mat}_{L+1}, \mathcal{L}_{3}^{2}+h\left(L(L+1)-\mathcal{L}^{2}\right)\right) .
$$

with $h>>1$. This picks out the fuzzy circle from the top angular momentum polarization tensor $\hat{Y}_{L, m}$.

One could equally pick it out from a lower one, $\hat{Y}_{L_{0}, m}$ by modifying the term proportional to $h$ to $\left(L_{0}\left(L_{0}+1\right)-\mathcal{L}^{2}\right)^{2}$. This latter choice may have advantages for the suppression of UV/IR mixing effects in the fuzzy context. It roughly corresponds to a mixture of 'nearest neighbour' and next nearest neighbour ferromagnetic and anti-ferromagnetic couplings.

Having constructed a fuzzy circle it is now clear that there is no obstacle to constructing fuzzy tori of arbitrary dimension, simply by taking products of fuzzy circles. This has the obvious advantage for numerical simulation of avoiding power-law curvature effects. 


\section{Approximating $S^{4}$ from $\mathbb{C P}_{F}^{3}$}

We can use a similar procedure to approximate $S^{3}$ from a finite approximation to $S^{4}$ but first, in this section, we summarise the construction of the fuzzy $S^{4}$ from fuzzy $\mathbb{C P}^{3}$ given in [4]. The construction utilises the fact that $\mathbb{C P}^{3}$ is an $S^{2}$ bundle over $S^{4}$ and there is a well-defined matrix approximation to $\mathbb{C P}^{3} \cong S U(4) / U(3)$. The harmonic expansion of a function on $\mathbb{C P}^{3}$ requires representations of $S U(4)$ that contain singlets of $U(3)$ under $S U(4) \rightarrow S U(3) \times U(1)$ : in terms of $S U(4)$ Young tableaux the permitted representations are

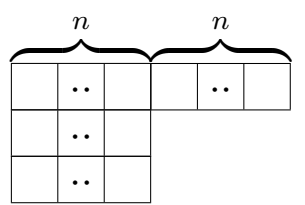

and are of dimension $\frac{1}{12}(2 n+3)(n+2)^{2}(n+1)^{2}$. All such representation, for $n \leq L$, appear in the tensor product

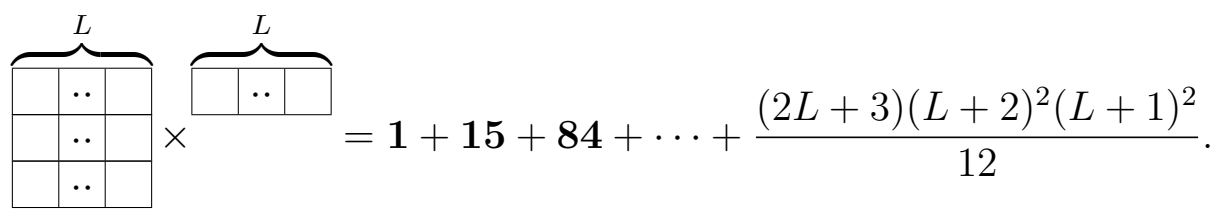

Since the dimension of $\overbrace{\square}^{L . \cdot}$ is $d_{L}:=\frac{1}{6}(L+3)(L+2)(L+1)$ the representations in (23) constitute a $d_{L} \times d_{L}$ matrix and are thus in one-to-one correspondence with elements $\Phi$ of $\mathrm{Mat}_{d_{L}}$.

Fuzzy $\mathbb{C P}^{3}$ is now identified with $\mathrm{Mat}_{d_{L}}$ with an appropriate Laplacian. The most natural Laplacian on $\mathbb{C P}_{F}^{3}$ is the $S U(4)$ invariant one which is the the second order Casimir corresponding to the adjoint action of the $S U(4)$ generators in the $d_{L} \times d_{L}$ representation. For future convenience we shall use the fact that $S U(4) \approx \operatorname{Spin}(6)$ and denote the generators by $J_{A B}$, with $A, B=1, \ldots, 6$ and $J_{A B}=-J_{B A}$. The $\operatorname{Spin}(6)$ invariant Laplacian on Mat $_{d_{L}}$ is then

$$
\mathcal{L}_{(6)}^{2} \Phi=\frac{1}{2}\left[J_{A B},\left[J_{A B}, \Phi\right]\right] .
$$

As $L \rightarrow \infty$ this corresponds to the continuum Spin(6) invariant Laplacian on $\mathbb{C P}^{3}$.

In the notation of [26] we shall label the $\operatorname{Spin}(6)$ irreducible representations by their highest weights $\left(n_{1}, n_{2}, n_{3}\right)$, with $n_{1}, n_{2}, n_{3}$ either all integers or all half-integers and $n_{1} \geq n_{2} \geq n_{3}$. The dimensions of these representations 
are

$d^{(6)}=\left(n_{1}, n_{2}, n_{3}\right)=\frac{1}{12}\left(\left(n_{1}+2\right)^{2}-\left(n_{2}+1\right)^{2}\right)\left(\left(n_{1}+2\right)^{2}-n_{3}^{2}\right)\left(\left(n_{2}+1\right)^{2}-n_{3}^{2}\right)$

and the eigenvalues of $\mathcal{L}_{(6)}^{2}$ are

$$
C_{2}^{(6)}\left(n_{1}, n_{2}, n_{3}\right)=\left(n_{1}\left(n_{1}+4\right)+n_{2}\left(n_{2}+2\right)+n_{3}^{2}\right) .
$$

The irreducible representations (22) that appear in a harmonic expansion of function on $\mathbb{C P}^{3}$ are $(n, n, 0)$ with $n$ an integer, so the quadratic Casimir takes the value

$$
C_{2}^{(6)}(n, n, 0)=2 n(n+3) .
$$

In this notation (23) translates to

$$
\overline{\left(\frac{L}{2}, \frac{L}{2}, \frac{L}{2}\right)} \times\left(\frac{L}{2}, \frac{L}{2}, \frac{L}{2}\right)=\sum_{n=0}^{L}(n, n, 0) .
$$

The extraction of a fuzzy $S^{4}$ from this algebra further relies on the curious fact that there is another possibility for the Laplacian on $\mathbb{C P}_{F}^{3}$ that has a lower symmetry, $S O(5)$, coming from the fact that it is also possible to represent $\mathbb{C P}^{3}$ as the coset space $S O(5) /(S U(2) \times U(1))$. In this representation the harmonic expansion of a function on $\mathbb{C P}^{3}$ requires all representations of $\operatorname{Spin}(5)$ that contain singlets of $S U(2) \times U(1)$ under the decomposition

$$
\begin{aligned}
\operatorname{Spin}(5) & \rightarrow S U(2) \times U(1) \\
\mathbf{4} & \rightarrow \mathbf{2}_{0}+\mathbf{1}_{1}+\mathbf{1}_{-1} \\
\mathbf{5} & \rightarrow \mathbf{2}_{1}+\mathbf{2}_{-1}+\mathbf{1}_{0} \\
\mathbf{1 0} & \rightarrow \mathbf{3}_{0}+\mathbf{2}_{1}+\mathbf{2}_{-1}+\mathbf{1}_{2}+\mathbf{1}_{-2}+\mathbf{1}_{0}
\end{aligned}
$$

Irreducible representations of $\operatorname{Spin}(5)$ can be labelled by two numbers $\left(n_{1}, n_{2}\right)$, either both integers or both half-integers, and $n_{1} \geq n_{2}$. They have dimension

$$
d^{(5)}\left(n_{1}, n_{2}\right)=\frac{1}{6}\left(n_{1}-n_{2}+1\right)\left(n_{1}+n_{2}+2\right)\left(2 n_{1}+3\right)\left(2 n_{2}+1\right)
$$

and second order Casimirs

$$
C_{2}^{(5)}\left(n_{1}, n_{2}\right)=\left(n_{1}\left(n_{1}+3\right)+n_{2}\left(n_{2}+1\right)\right) .
$$

From (29) we see that the Spin(5) representations that contain singlets of $S U(2) \times U(1)$ are those with $\left(n_{1}, n_{2}\right)$ both integers - these are all the tensor 
representations $T_{a_{1} \cdots a_{n}}$, with $a_{j}=1, \ldots 5$, and are therefore really representations of $S O(5)$. The $S O(6)$ representations appearing in (23) decompose into $S O(5)$ representations as

$$
\begin{aligned}
S O(6) & \rightarrow S O(5) \\
\mathbf{1 5} & \rightarrow \mathbf{5}+\mathbf{1 0} \\
\mathbf{8 4} & \rightarrow \mathbf{1 4}+\mathbf{3 5}+\mathbf{3 5 ^ { \prime }}
\end{aligned}
$$

or, in general,

$$
(n, n, 0) \rightarrow \sum_{m=0}^{n}(n, m) .
$$

The fact that $\mathbb{C P}^{3} \cong S O(5) /(S U(2) \times U(1))$ means that $S O(5)$ acts transitively on $\mathbb{C P}^{3}$ and functions on $\mathbb{C P}^{3}$ can be expanded in terms of $S O(5)$ irreducible representations $(n, m)$ with an $S O(5)$ invariant Laplacian. As discussed in [4], there is no unique $S O(5)$ invariant Laplacian on $\mathrm{Mat}_{d_{L}}$ but rather any linear combination of the restrictions of (24) to $S O(5)$ : i.e. any linear combination of

$$
\mathcal{L}_{(5)}^{2} \Phi=\frac{1}{2}\left[J_{a b},\left[J_{a b}, \Phi\right]\right]
$$

and

$$
\mathcal{L}_{(v)}^{2} \Phi=\left[J_{a},\left[J_{a}, \Phi\right]\right],
$$

with $a, b=1, \ldots, 5$ and where $J_{a}=J_{a 6}$, can be used as a Laplacian provided the combination has positive eigenvalues.

The fuzzy $S^{4}$ can now be extracted from this by noting that the harmonic expansion of functions on $S^{4} \cong S O(5) / S O(4)$ require irreducible representations of $S O(5)$ that contain singlets of $S O(4)$ under the restriction of $S O(5)$ to $S O(4)$. These are of course the symmetric tensor representations of $S O(5)$, labelled by $(n, 0)$ in the notation above. A Laplacian whose low lying modes are those of $S_{F}^{4}$ can be constructed by penalizing the modes $(n, m)$ in $(33)$ with $m \neq 0$. From (27) and (31) we see that

$$
2 C_{2}^{(5)}(n, m)-C_{2}^{(6)}(n, n, 0)=2 m(m+1),
$$

so the Laplacian

$$
\mathcal{L}_{h}^{2}=\mathcal{L}_{(6)}^{2}+h\left(2 \mathcal{L}_{(5)}^{2}-\mathcal{L}_{(6)}^{2}\right)
$$

has eigenvalues $2 n(n+3)+2 h m(m+1)$ and states with $m>0$ will be suppressed in a functional integral for large $h$. The parameter $h$ here is acting like a "squashing" parameter, $h=0$ is the "round" $S O(6)$ invariant metric on $\mathbb{C P}^{3}$, while $h \neq 0$ breaks this symmetry down to $S O(5)$. The lowest 
permitted value for $h$ is $h=-1$. We have $\mathcal{L}_{-1}^{2}=2 \mathcal{L}_{v}^{2}$, and we see that this Laplacian is rather singular in the large $L$ limit as the representation $(n, n)$ for large $n$ develops a zero eigenvalue.

The family of actions

$$
S_{h}[\Phi]=\frac{1}{d_{L}} \operatorname{Tr}\left\{\Phi^{\dagger} \mathcal{L}_{h}^{2} \Phi+V(\Phi)\right\}
$$

gives a field theory on squashed $\mathbb{C P}_{F}^{3}$ for $h>-1$. Furthermore as $h \rightarrow \infty$ modes with $m>0$ are completely suppressed in a functional integral and (38) corresponds to a field theory on $S_{F}^{4}$. Note that it does not matter whether we use $\mathcal{L}_{(6)}^{2}, 2 \mathcal{L}_{(5)}^{2}$ or $2 \mathcal{L}_{(v)}^{2}$ for the first term on the right-hand side of (37) when the constraint $m=0$ is imposed all three become the same operator.

\section{$5 \quad S_{F}^{3}$ from $S_{F}^{4}$}

We can build on the construction of the last section to get a Laplacian whose low lying modes are those associated with a field on $S_{F}^{3}$ by using the same trick as in section 3 to pick out the top mode $n=L$ of the $S_{F}^{4}$. As an irreducible representation of $S O(5)$ this is the representation $(L, 0)$ with dimension

$$
d^{(5)}(L, 0)=\frac{1}{6}(2 L+3)(L+2)(L+1)
$$

The harmonic expansion of a function on $S^{3} \cong S U(2)$ requires all irreducible representations of $S U(2)$, both integral and half-integral,

$$
f_{L}(\theta, \phi, \psi)=\sum_{j=0, \frac{1}{2}, \ldots}^{L / 2} \sum_{m=-j}^{j} \sum_{m=-j}^{j} f_{\bar{m}, m}^{j} D_{\bar{m}, m}^{j}(\theta, \phi, \psi),
$$

where $(\theta, \phi, \psi)$ are Euler angles and $D^{j}(\theta, \phi, \psi)$ are the Wigner $D$-matrices. The key to extracting $S_{F}^{3}$ from $S_{F}^{4}$ is the observation that the total number of degrees of freedom in (40) is

$$
\sum_{j=0, \frac{1}{2}, \ldots}^{L / 2}(2 j+1)^{2}=\frac{1}{6}(2 L+3)(L+2)(L+1)
$$

which is the same as $d^{(5)}(L, 0)$ in (39). This is because the top mode (or indeed any mode $(n, 0))$ of $S_{F}^{4}$ has the representation content of an $S_{F}^{3}$. 
The top $S O(5)$ mode of $S_{F}^{4}$ can now be picked out by penalising the modes with $n<L$ with an 'anti-ferromagnetic' kinetic-energy term. To this end we define the Laplacian

$$
\mathcal{L}_{h, h^{\prime}}^{2} \Phi=\frac{1}{2}\left[J_{\alpha \beta},\left[J_{\alpha \beta}, \Phi\right]\right]+h^{\prime}\left(2 L(L+3)-\mathcal{L}_{(6)}^{2}\right) \Phi+h\left(2 \mathcal{L}_{(5)}^{2}-\mathcal{L}_{(6)}^{2}\right) \Phi
$$

with $\alpha, \beta=1, \ldots, 4$. For finite $h$ and $h^{\prime}$, as both the last two terms are $>0$, this is a positive operator and contains all modes on $\mathbb{C P}_{F}^{3}$. As $h \rightarrow \infty$ modes not relevant to $S_{F}^{4}$ are sent to infinity and, finally, modes not relevant to $S_{F}^{3}$ are sent to infinity when $h^{\prime} \rightarrow \infty$. For very large $h$ and $h^{\prime}$, the low lying eigenvalues are therefore precisely those of $S_{F}^{3}$. In a functional integral for a scalar field based on this Laplacian we recover scalar field theory on $S_{F}^{3}$ in the large $h$ and $h^{\prime}$ limit. Field theory on $S_{F}^{3}$ therefore arises from the double limit $h, h^{\prime} \rightarrow \infty$ in the action

$$
S_{h, h^{\prime}}[\phi]=\frac{1}{d_{L}} \operatorname{Tr}\left\{\Phi^{\dagger} \mathcal{L}_{h, h^{\prime}}^{2} \Phi+V(\Phi)\right\}
$$

with $\Phi \in \operatorname{Mat}_{d_{L}}$.

\section{$6 \quad$ An alternative construction of $S_{F}^{3}$}

The constructions described up till now have relied on matrix approximations to $\mathbb{C P}^{N}$, specifically $\mathbb{C P}^{1} \cong S^{2}$ and $\mathbb{C P}^{3}$. There is however another construction for $S_{F}^{3}$ based on the orthogonal Grassmannian $S O(5) /(S O(3) \times S O(2))$. This is a co-adjoint orbit and therefore a well-defined finite matrix approximation to the algebra of functions on this Grassmannian exists. This space is not the same as $\mathbb{C P}^{3}$ : it arises from a different embedding of $S U(2) \times U(1)$ into $\operatorname{Spin}(5)$, characterised by the decomposition

$$
\begin{aligned}
\operatorname{Spin}(5) & \rightarrow S U(2) \times U(1) \\
\mathbf{4} & \rightarrow \mathbf{2}_{1}+\mathbf{2}_{-1} \\
\mathbf{5} & \rightarrow \mathbf{3}_{0}+\mathbf{1}_{2}+\mathbf{1}_{-2} \\
\mathbf{1 0} & \rightarrow \mathbf{3}_{2}+\mathbf{3}_{-2}+\mathbf{3}_{0}+\mathbf{1}_{0} .
\end{aligned}
$$

In particular the $\mathbf{5}$ does not give a singlet under this embedding and so must be excluded from the harmonic expansion on this space - it is clearly not the same space as $\mathbb{C P}^{3}$. The representation content here is such that

$$
S O(5) /(S O(3) \times S O(2)) \cong S p(2) / U(2),
$$


which is known not to admit a spin structure [27]. The expansion of a function on the orthogonal Grassmannian $S O(5) /(S O(3) \times S O(2))$ can be obtained from that on $\mathbb{C P}^{3} \cong S O(5) /(S U(2) \times U(1))$ simply by omitting all the odd rank tensors from the latter. In this way $S O(5) /(S O(3) \times S O(2))$ follows from moding out the $S O(5)$ representation of $\mathbb{C P}^{3}$ by the $\mathbb{Z}_{2}$ action $T_{a}-\rightarrow-T_{a}$ on the 5 , so

$$
S O(5) /(S O(3) \times S O(2)) \cong \mathbb{C P}^{3} / \mathbb{Z}_{2}
$$

As a side remark we note that $S O(5) /(S O(3) \times S O(2))$ is an $S^{2}$ bundle over the real projective space $\mathbb{R P}^{4}$.

In the notation of section 4 the even rank tensor representations of $S O(5)$ are $(n, m)$ with $n+m=2 l$, and these have dimension

$$
d^{(5)}(2 l-m, m)=\frac{1}{6}(2 l-2 m+1)(2 l+2)(4 l-2 m+3)(2 m+1) .
$$

So the total number of degrees of freedom for $m \leq l$ and $0 \leq l \leq L / 2(L$ even) is

$$
\sum_{l=0}^{L / 2} \sum_{m=0}^{l} d^{(5)}(2 l-m, m)=\left[\frac{(L+4)(L+3)(L+2)}{24}\right]^{2}
$$

which is the same as that of Mat $_{d_{L}^{\prime}}$ with

$$
d_{L}^{\prime}=\frac{1}{24}(L+4)(L+3)(L+2) \text {. }
$$

Thus for matrix dimensions $d_{L}^{\prime}$ we have fuzzy orthogonal Grassmanians. The action on this fuzzy Grassmannian looks essentially identical to that on a squashed $\mathbb{C P}_{F}^{3}$,

$$
S[\Phi]=\frac{1}{d_{L}^{\prime}} \operatorname{Tr}\left\{\Phi^{\dagger} \mathcal{L}_{(5)}^{2} \Phi+V(\Phi)\right\},
$$

except that the matrix algebras are restricted to those of size $d_{L}^{\prime}$ in (49) containing only even rank tensor representations $S O(5), d^{(5)}(2 l-m, m)$ and one has only one quadratic Casimir, the $S O(5)$ one, at ones disposal.

The harmonic expansion of a function on $S_{F}^{3}$ is contained in Mat $d_{L}^{\prime}$ because the top representation for a given $L, 2 l=L$ with $m=0$, has dimension (39) and, as observed in the previous section, this is a sum of the dimensions of the $S U(2)$ representations required for a harmonic expansion on $S^{3}$, (41). 
If we can penalise all modes with $2 l<L$ and $m \neq 0$ for $2 l=L$ in a functional integral over $S O(5) /(S O(3) \times S O(2))$ then we will really be doing a functional integral over $S_{F}^{3}$. This is easily achieved since $2 l=L$ and $m=0$ has the largest second order Casimir,

$$
C_{2}^{(5)}(L, 0)=L(L+3)
$$

of all the $S O(5)$ representations in $\mathrm{Mat}_{d_{L}^{\prime}}$. In the now familiar manner the unwanted modes in the functional integral over $S O(5) /(S O(3) \times S O(2))$ can be suppressed by using the Laplacian

$$
\mathcal{L}_{h^{\prime}}^{2}=\frac{1}{2}\left[J_{\alpha \beta},\left[J_{\alpha \beta}, \cdot\right]\right]+h^{\prime}\left(L(L+3)-\mathcal{L}_{(5)}^{2}\right),
$$

which acts on fields $\Phi \in \mathrm{Mat}_{d_{L}^{\prime}}$ and $L$ even. The unwanted modes are completely eliminated in the limit $h^{\prime} \rightarrow \infty$, giving $S_{F}^{3}$ truncated at level $L$. The constraint that $L$ is even does not change the fact that we get the full continuum $S^{3}$ as $L \rightarrow \infty$.

\section{Conclusions}

By starting with the known finite matrix algebras for $\mathbb{C P}^{3}$ and $\mathbb{C P}^{1}$, the fuzzy $\mathbb{C P}_{F}^{3}$ and the fuzzy sphere $\mathbb{C P}_{F}^{1} \cong S_{F}^{2}$, finite functional integrals for scalar field theories on $S_{F}^{3}$ and $S_{F}^{1}$ have been constructed. The geometry of a fuzzy space is specified by a triple $\left(H_{L}\right.$, Mat $\left._{d_{L}}, \Delta_{L}\right)$ and, although there is no known closed associated algebra giving a fuzzy $S^{1}$ as a triple directly, $\mathbb{C P}_{F}^{1}$ nevertheless contains the states required for a $S_{F}^{1}$ plus other unwanted states. The unwanted states are given large eigenvalues by modifying the Laplacian on $\mathbb{C P}_{F}^{1}$, as in equation (21), leaving only the states of $S_{F}^{1}$ in the low energy spectrum of the Laplacian. In a similar way $\mathbb{C P}_{F}^{3}$ contains the states necessary for a fuzzy description of $S^{3}$ (via $S_{F}^{4}$ ) and the Laplacian on $\mathbb{C P}_{F}^{3}$ can be modified, as in equation (42), so that states not related to $S_{F}^{3}$ are given large eigenvalues, leaving only $S_{F}^{3}$ states in the low energy spectrum.

An alternative construction of $S_{F}^{3}$, based on suppressing modes on a fuzzy version of the orthogonal Grassmannian $S O(5) /(S O(3) \times S O(2))$, has been presented in section 6 . This has the advantage of having a natural extension to $S_{F}^{N}$ for any $N,[20]$. Thus $S_{F}^{3}$ can be obtained either in two steps, via the fuzzy $S_{F}^{4}$ constructed in [4], $\mathbb{C P}_{F}^{3} \rightarrow S_{F}^{4} \rightarrow S_{F}^{3}$, or alternatively in a single step from the fuzzy version of $S O(5) /[S O(3) \times S O(2)]$ as described in section 6. 
The construction of the fuzzy circle allows fuzzy tori to be defined in an obvious way, by taking products of fuzzy circles, thus opening the way to numerical simulations on tori while preserving the full $U(1) \times \cdots \times U(1)$ isometry group and avoiding the fermion doubling problem [5]. One first writes down a finite functional integral for a field theory on $S_{F}^{2} \times \cdots \times$ $S_{F}^{2}$, which contain the modes relevant for propagation $S_{F}^{1} \times \cdots \times S_{F}^{1}$ in its spectrum, and then damps the unwanted modes. This can be done, in a manner that preserves the isometries of the torus, by introducing appropriate combinations of second order Casimirs into the propagators.

It is pleasure to acknowledge A. P. Balachandran, Peter Prešnajder, Harald Grosse and Daniel Roggenkamp for helpful discussions.

\section{References}

[1] I. Montvay and G. Munster, Quantum Field on a Lattice, Cambridge, 1997.

[2] S. Vaidya and B. Ydri, On the Origin of the UV-IR Mixing in Noncommutative Matrix Geometry, hep-th/0305201.

[3] G. Alexanian, A.P. Balachandran, G. Immirzi and B. Ydri, J. of Geom. and Phys. 42 (2002) 28, hep-th/0103023.

[4] Denjoe O'Connor and Julieta Medina, Scalar Field Theory on Fuzzy $S^{4}$, hep-th/0212170.

[5] A.P. Balachandran, T.R. Govindarajan and B. Ydri, Mod. Phys. Lett. A15 (2000) 1279, hep-th/9911087; A.P. Balachandran, T.R. Govindarajan and B. Ydri, Fermion doubling problem and noncommutative geometry II, hep-th/0006216; A.P. Balachandran and G. Immirzi, The Fuzzy Ginsparg-Wilson Algebra: A Solution of the Fermion Doubling Problem, hep-th/0301242.

[6] A.P. Balachandran, S. Kurkcuoglu and E. Rojas, JHEP 0207 (2002) 056, hep-th/0204170.

[7] A. Yu. Alekseev, A. Recknagel and V. Schomerus, JHEP 9909, (1999) 023, hep-th/9908040.

[8] Y. Hikida, M. Nozaki and Y. Sugawara, Nucl. Phys. B617, (2001) 117, hep-th/0101211. 
[9] H. Grosse, C. Klimčík and P. Prešnajder, Int. J. Theor. Phys. 35, (1996) 231, hep-th/9505175; H. Grosse and A. Strohmaier, Lett. Math. Phys. 48, (1999) 163, hep-th/9902138.

[10] H. Grosse, C. Klimčík and P. Prešnajder, Comm. Math. Phys. 178, (1996) 507; H. Grosse and P. Prešnajder, Lett. Math. Phys. 46, (1998) 61.

[11] P. Prešnajder, J. Math. Phys. 41 (2000) 2789, hep-th/9912050.

[12] A.P. Balachandran and S. Vaidya, Int. J. Mod. Phys. A16, (2001) 17, hep-th/9910129; S. Baez, A.P. Balachandran, S. Vaidya and B. Ydri, Comm. Math. Phys. 208, (2000) 787, hep-th/9811169.

[13] J. Madore, Class. Quant. Grav. 9 (1992) 69.

[14] S. Ramgoolam, Nucl. Phys. B610 (2001) 461, hep-th/0105006.

[15] Yusuke Kimura, On higher dimensional fuzzy spherical branes, hep-th/0301055.

[16] S. Ramgoolam, JHEP 0210 (2002) 064, hep-th/0207111.

[17] P.M. Ho and S. Ramgoolam, Nucl.Phys. B627 (2002) 266, hep-th/0111278.

[18] A.P. Balachandran, B.P. Dolan, J. Lee, X. Martin and D. O'Connor, J. Geom. and Phys. 43 (2002) 184, hep-th/0107099.

[19] J. Frohlich and K. Gawędzki, Conformal Field Theory and Geometry of Strings, Lectures given at Mathematical Quantum Theory Conference, Vancouver, Canada, 4-8 Aug 1993. Published in Vancouver 1993, Proceedings, Mathematical quantum theory, Vol. 1 57-97, hep-th/9310187.

[20] B.P. Dolan, Denjoe O'Connor and P. Prešnajder, in preparation.

[21] A. Connes. Noncommutative Geometry, Academic Press, London, 1994.

[22] F.A. Berezin, Comm. Math. Phys. 40, (1975) 153.

[23] B.P. Dolan, Denjoe O'Connor and P. Prešnajder, JHEP 0203 (2002) 013, hep-th/0109084.

[24] D.A. Varshalovich, A.N. Moskalev and V.K. Khersonskii, Quantum Theory of Angular Momentum, World Scientific (1988) 
[25] Chong-Sun Chu, J. Madore and H. Steinacker, JHEP 0108 (2001) 038, hep-th/0106205.

[26] W. Fulton and J. Harris , Representation Theory: a first course, Graduate Texts in Mathematics 129, Springer, (1991).

[27] B.P. Dolan and C. Nash, JHEP 0207 (2002) 057, hep-th/0207007. 